\title{
NEW REALITIES FOR CANADA'S PARLIAMENT: A WORKFLOW FOR PREPARING HERITAGE BIM FOR GAME ENGINES AND VIRTUAL REALITY
}

\author{
C. Pybus ${ }^{1 *}$, K. Graham ${ }^{1 *}$, J. Doherty ${ }^{2}$, N. Arellano ${ }^{1}$, S. Fai ${ }^{1}$ \\ ${ }^{1}$ Carleton Immersive Media Studio, Carleton University, 1125 Colonel By Drive Ottawa, Canada - (cpybus, kgraham, narellano \\ sfai)@cims.carleton.ca \\ ${ }^{2}$ Azrieli School of Architecture and Urbanism, Carleton University - joeyrdoherty@ cmail.carleton.ca
}

Commission II, WG II/8

KEY WORDS: HBIM, VR, virtual reality, digital cultural heritage, game engines, serious games, storytelling

\begin{abstract}
:
With a growing interest in the use of virtual reality (VR) for dissemination of cultural heritage sites, the question of how to leverage existing documentation as content for virtual experiences becomes a potentially valuable opportunity. Notably, as sites are increasingly documented with building information modelling (BIM) for the purposes of conservation, there is potential to give these models a second life as content for public education and promotion. However, although software exist for viewing BIM in VR headsets, they are inadequate for complex models typical of heritage buildings, and lack functionality for integrating custom didactic content and storytelling. To make BIM performative in VR and allow for custom content, a workflow was developed to translate BIM into game engine scenes - which optimizes geometry following performance guidelines of VR while maintaining the high visual fidelity of the BIM. As a case study, six heritage spaces of the Centre Block of the Canadian Parliament which had been previously documented and modelled by CIMS were prepared for Unity3D, enabling their later use in a storytelling experience.
\end{abstract}

\section{INTRODUCTION}

The collection of digital heritage recordings presents new opportunities to use this burgeoning archive for unexplored applications. This paper demonstrates a workflow for adapting a heritage BIM of Canada's Parliament for use in game engines and viewing in virtual reality. Since late 2018, the Centre Block of Canada, the centrepiece of the Parliament Hill National Historic Site in the country's capital, Ottawa, is undergoing a multi-year rehabilitation project that has recently seen the closure of the iconic building to all public tours and parliamentary activities. Carleton Immersive Media Studio (CIMS), a research unit associated with the Azrieli School of Architecture and Urbanism at Carleton University in Ottawa, Canada, has had the opportunity to be involved since 2013 in the documentation and modelling of existing conditions of the Parliament Buildings and grounds as well as its dissemination for public outreach. A building information model (BIM) of all prominent buildings and the grounds has been created by converting the terrestrial laser scanning metric data into a highly detailed and accurate representation in Autodesk Revit. To aid in public dissemination, CIMS translated the comprehensive BIM into an accessible Virtual Reality (VR) experience, explorable through the game engine Unity 3D and the Oculus Rift VR headset. Although CIMS participated only in the creation of these VR scenes, they were prepared to accommodate storytelling content to be produced at a later time.

Even though the BIM contained a level of detail appropriate for visualizations and renderings, the data was not easily converted into Unity 3D for VR due to the complexity of geometry, unoptimized meshes, and unrealistic textures. CIMS devised a workflow using various software to help convert the BIM into a workable VR experience that meets the criteria outlined by Oculus Rift for optimized performance. This paper will provide an overview of the workflow to convert BIM to VR for a publicly accessible experience and discuss the issues that arose through its development.

\section{DOCUMENTATION AND MODELLING}

The site, comprising of the Library of Parliament, Centre Block, West Block, East Block, and grounds was originally constructed beginning in 1859 . It quickly became a symbol of Canadian parliamentary democracy and admired for its exemplary Gothic Revival style. After a fire destroyed the original Centre Block in 1916, a reconstruction began designed by John A. Pearson and Jean Omer Marchand. The new building showcased cutting edge technologies for construction at the time - such as a new steel structural system - while retaining the ornamental gothic revival quality of the other buildings still standing. A comprehensive rehabilitation project began on Parliament Hill in 2002 intending to repair the historic fabric, modernizing services, and addressing changes in its functional program. The Centre Block program of work began late 2018. Tours of the Centre Block are suspended for the duration of construction with alternate means of engaging with the public, both on-site and remotely, being studied. These include virtual reality and other digitally assisted storytelling methods.

In partnership with Heritage Conservation Services (HCS), a division of Public Services and Procurement Canada (PSPC), the grounds and buildings of the Parliament Hill National Historic Site were documented using a combination of terrestrial laser scanning and photogrammetry. More than 2000 individual scan stations captured three terabytes of data. All significant heritage spaces in the Centre Block, such as the House of Commons and Senate Chamber, were documented using photogrammetry by HCS while additional smaller assets, such as carvings, were captured by CIMS on request.

\footnotetext{
* Corresponding authors
} 


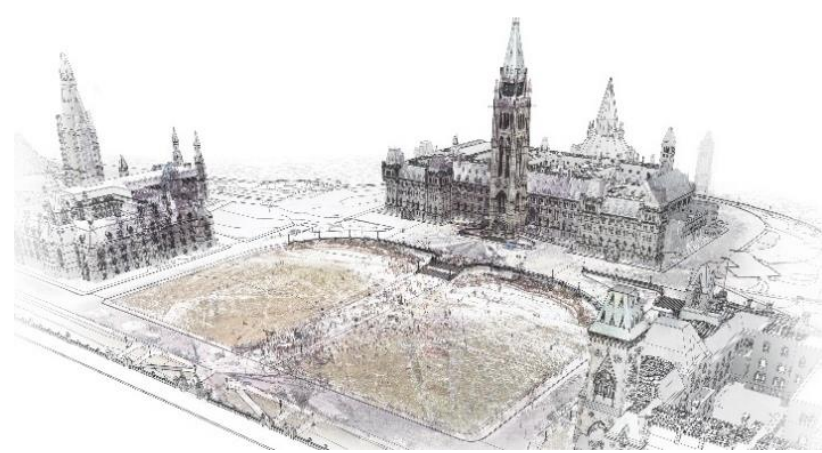

Figure 1. BIM Parliament Hill with point cloud overlaid.

The data acquired through laser scanning and photogrammetry, in addition to historic construction drawings and photographs from Library and Archives Canada, were used by CIMS to create a highly detailed Building Information Model (BIM) of the site in Autodesk Revit (figure 1). The purpose of the documentation and model was to assist in the design and construction management of the rehabilitation project and serve as a historic record of the iconic heritage buildings.

Specialty spaces in the Centre Block, including the House of Commons and Senate chambers and foyers, the Rotunda, and the Library of Parliament (figure 2), were modelled at a higher level of detail to ensure their heritage value was recorded and archived. As 3D models, these spaces have the potential to be used directly for purposes beyond an archive, such as public dissemination in computer-generated images, videos, and interactive experiences. The six spaces became the area of study for converting the BIM into a working VR experience using Unity 3D.

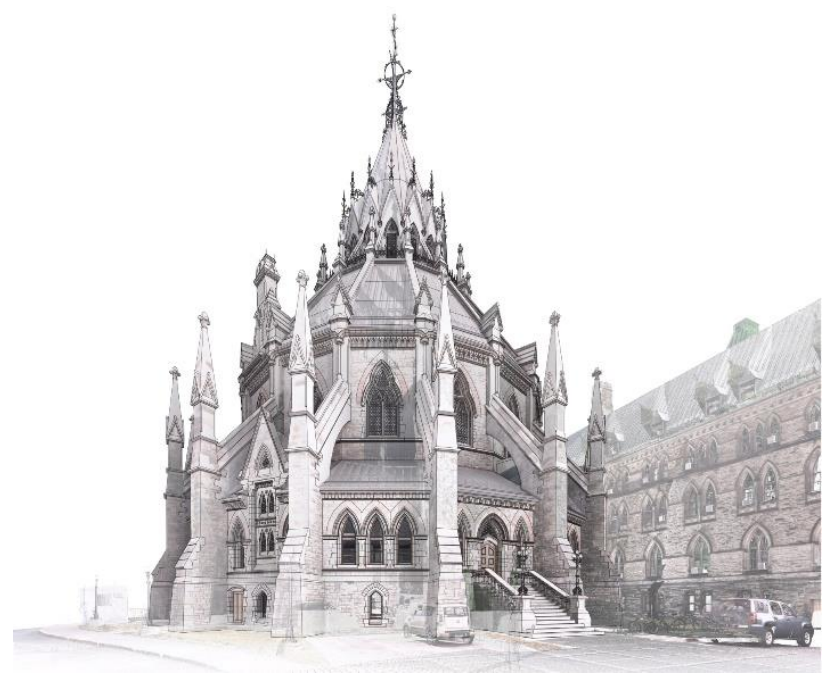

Figure 2. BIM with point cloud of the Library of Parliament.

\section{STATE OF THE ART}

\subsection{Advantages of Virtual Reality}

The classifying trait of VR is the sense of presence - the feeling of 'being there.' A term first introduced as telepresence by Marvin Minsky about teleoperations and the control of remote robots (Minsky, 1980), presence later translated to describe the feeling and belief of being present in a virtual world (Held and Durlach, 1992; Sheridan, 1992; Slater and Usoh, 1993; Steuer, 1992; Zeltzer, 1992). According to researchers, presence is achieved when the virtual world behaves consistently, avoids interference from external elements, and contains a correlation between the user's physical and virtual movements. With VR and architecture both relating to the occupying of space, VR's use for architecture is promising, with three decades of research. Initial research into VR's benefit for architecture occurred with the first VR research boom in the 1980s and 1990s. Led by Dr. Frederick Brooks, the VR Laboratory in the Department of Computer Science at the University of North Carolina at Chapel Hill researched VR's application in medical imaging to architecture (Rheingold, 1991). As an initial prototype into the benefits of VR for architecture, the lab created a simulation of the University's Computer Science building they were located in to create a tool beneficial for communication between architects and clients (Brooks, 1987). The virtual environment was accessible through a large head-mounted display that transported the wearer to a virtual twin of the building they physically occupy.

Due to limitations in VR technology, the research boom of the 1980s and 1990s did not convert into commercially viable applications. Only recently, when in 2012 the Oculus Rift Kickstarter launched and in 2016 the first commercial headsets of the Oculus Rift, HTC Vive, and Playstation VR were released, has the benefits of VR for the architectural and heritage fields been revisited.

\subsection{Virtual Reality for Cultural Heritage}

The use of VR to assist in the dissemination of heritage architecture through public outreach is found in projects that strive to bring the public into spaces that are not accessible due to unsafe conditions or temporal destructions. In his 1998 book, Rendering Real and Imaged Buildings: the Art of Computer Modeling from the Palace of Kublai Khan to Le Corbusier's Villas, B.J. Novitski outlines the importance of using virtual reproductions of historic buildings for both public dissemination and research. Technology brings the history of place alive through walkable, visually stimulating digital reconstructions (Novitski, 1998). Previous work outlines advantages of using game engines for cultural heritage dissemination and demonstrates its application in storytelling (Pybus 2018).

One method for documenting and virtually reconstructing cultural heritage sites uses photogrammetry. Photogrammetry uses the combination of multiple photographs of an object to extract measurements and create an accurate three-dimensional digital model. The photogrammetric model created is limited to only include what was captured by the camera. If a portion is missed in the documentation process, such as the top or small crevice in a corner, the model will contain inaccuracies; however, if captured in entirety, the model can offer a high-fidelity digital replica of the physical asset. Lachambre et al. discuss how the meshes created with the documentation process can be added to game engine technology and virtual reality experiences (Lachambre et al., 2017). Dhanda et al., through the case study of the Myin-pya-gu $11^{\text {th }}$ century temple in Bagan, Myanmar, develop a process for optimizing the meshes using texture maps, normal maps, ambient occlusion maps, roughness maps, and metallic maps (Dhanda et al., 2019); and previous research provides an overview of viable game engines for cultural heritage and examines the accessibility of VR experiences for tourism (Pybus 2019).

\subsection{Virtual Reality for BIM}

The benefits of VR for the AEC industry, especially for design and communication with the project stakeholders, are a reason for a multitude of one-click conversion software to be created. Software, such as Advent Twinmotion, Enscape, Iris VR promise 
the quick and simple translation from BIM to viewable and interactive VR model. The target market for these products is AEC professionals not interested in dealing with technical issues related to VR applications and desiring to quickly be able to enter the model for design or visualizations. Unfortunately, due to the simplicity of the software, limitations exist preventing the tool to be used for the conversion of a complex BIM for public dissemination. Through testing the available software with the Library of Parliament model, it was determined that they were inadequate for the creation of a VR space intended for public outreach. The software allowed for the viewing of the model, but not further development into a comprehensive storytelling project. Testing of the software confirmed literature analysis that most existing conversions of BIM to VR are used solely for the design phase and to aid in communication with stakeholders (Du et al., 2018; Zaker and Coloma, 2018).

As an alternative to the one-click BIM to VR methods, research into the challenge of adapting BIM to VR for public outreach common in cultural heritage suggests the use of a game engine to make VR accessible. The advantages of importing CAD models into game engines and the associated challenges of data interoperability were demonstrated as early as 2002 (Lehtinen, 2002). The development of a post-processing procedure called a 'crossover module,' as theorized by Yan et al., helps overcome these challenges by facilitating the conversion from BIM to VR (Yan et al., 2011). Additionally, through an exhaustive examination of existing case studies, Bille et al. identify the need for a post-processing workflow (Bille et al., 2018).

In a study of how to create bi-directional data exchange, Nandavar et al., discuss the importance of VR to the AEC industry for providing a heightened understanding of space, materials, and textures. Their study identifies the issues of interoperability between BIM and VR (Nandavar et al., 2018) and addresses the critical issues of data performance, memory loss, and remapping of textures common in the research (Eliashvili, 2016). The conversion of the Centre Block BIM to VR saw similar problems, which the new methodology sought to overcome.

As shown in the previous research, when converting BIM to VR for a curated experience, the common issues that arise are related to the complexity of the BIM and its poor translation in mesh geometry and texture. To solve this problem, the previous researchers have identified a need for a crossover module - or method to convert the BIM data into VR - that organizes and optimizes the meshes. Without a rigorous workflow, the result is a poorly organized and low fidelity VR experience.

\section{HERITAGE BIM TO VR WORKFLOW}

The primary specification used for determining the outputs of this process were taken from Oculus' publically available development guide (Oculus Documentation, n.d.). It recommends that for displaying an environment in an Oculus Rift the total triangular polyfaces should not exceed two million, and the number of draw calls - which describe how many objects are rendered to the screen per frame - should not exceed 1,000. Respecting both of these constraints helps to guarantee the frame rate of the headset remains at a stable 90 frame per second, which is essential for a comfortable VR experience. The workflow developed to create an optimized scene is shown in figure 5.

\subsection{Creating Meshes from BIM}

4.1.1 Export from Revit: Notably, Revit stores geometry as solid-body objects rather than meshes, and does not provide control of meshing parameters at export. Observing that Revit is lacking in mesh functions, it was preferred to export geometry to another software first as solid-body geometry, to allow better control over mesh optimization while retaining the accuracy of the BIM. The model was first divided into the six target spaces the House of Commons and Senate chambers and foyers, the Rotunda, and the Library of Parliament (figure 3), and exported from Revit in .DWG solid-body format, with specific high-detail parts exported as .SAT. Given the huge dataset of the parliament BIM, however, it was fundamentally necessary to develop a strategy of dividing the model into manageable parts.

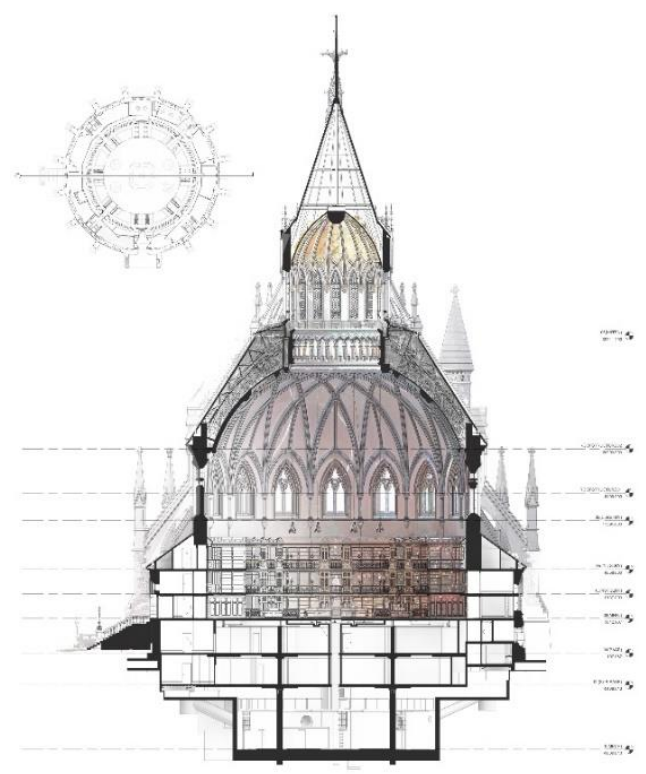

Figure 3. Revit model of the Library of Parliament.

4.1.2 Dividing the Model: A heritage space within the Revit model was split into several parts upon export in accordance with Revit family categories. This strategy provided advantages in that certain family categories require inherently more or less optimization in subsequent steps, and therefore dividing by family categories constituted a pre-filtering of geometry requiring optimization, and ensured that files were small enough to be used effectively. This division strategy, detailed in another publication as 'Geometry Levels (Graham et al., n.d.),' was further refined in relation to conventional architectural levels of detail. Revit elements were split into one of four Geometry Levels, based on their role in representing the space's character (figure 4). Larger, room-defining elements such as walls were classified as a Geometry Level 1 while smaller, ornamental elements such as cornices were classified as Geometry Level 4. A dynamo script was created so pre-filtering could be completed semi-automatically, saving on labour.
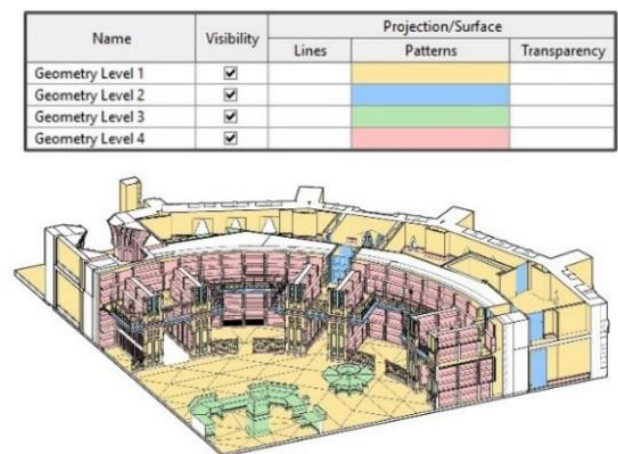

Figure 4. Revit model divided into four Geometry Levels. 


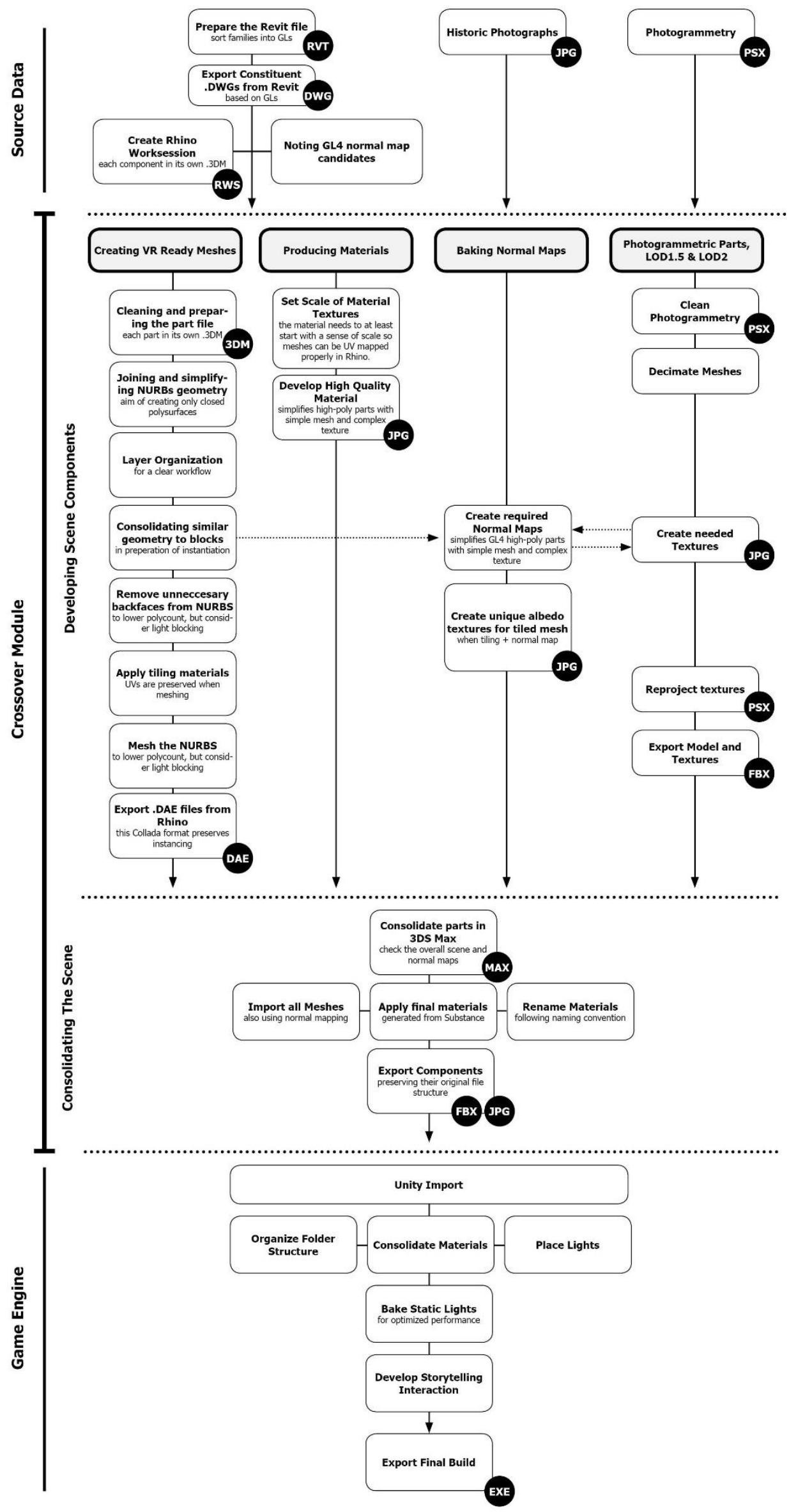

Figure 5. Overall workflow for exporting BIM, images, and photogrammetry into the Unity Game Engine. Note that some process can develop in parallel. 
4.1.3 Inspection, Repair, and Meshing in Rhinoceros 3D: Rhinoceros 3D was selected as the preferred software for converting solid-body geometry into meshes because of existing software knowledge among lab staff and compatibility between solid-body types, NURBS, and meshes. Overall this step involved importing a single. DWG set into Rhinoceros 3D, correcting errors in the import process (figure 6), organizing common elements into block instances, meshing the whole set, and exporting with the .DAE Collada format. In some cases it was necessary to rebuild the topology of certain NURBS objects, which was one of the more manual steps of the process which could be improved with better Revit export functionality. Block instances for repeating geometry were essential for minimizing draw calls within Unity. The .DAE format was used because it was the only mesh format available in Rhino which preserved the instantiated characteristic of geometry upon export.

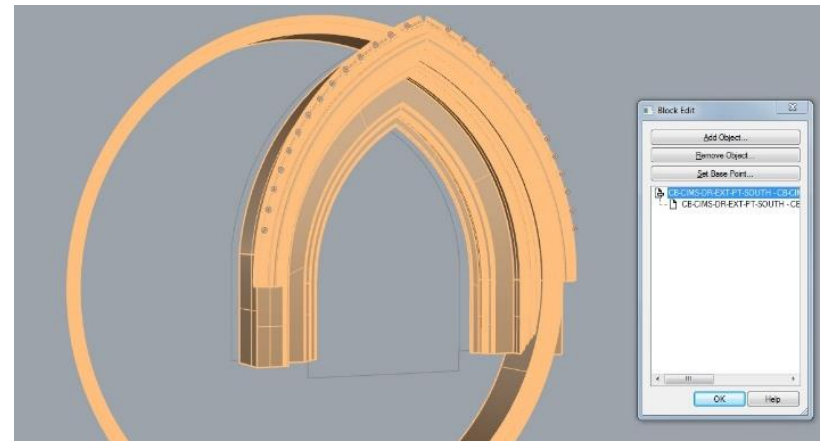

Figure 6. Correcting model errors from the export process.

4.1.4 Making Game-Ready in 3DS Max: In Autodesk 3DS Max, final preparations of the component were made to prepare it for Unity $3 \mathrm{D}$, converting the file from .DAE to a final gameready .FBX format. This step was of particular importance to ensure several mesh characteristics: minimized total mesh components, correct block instancing (figure 7), correct UV space, correct naming conventions, applying smoothing groups, and consolidated material assignments. This step was crucial for optimizing 'draw calls.' For example a single geometry instanced six times is considered a single draw call rather than six. A companion constraint to polycount, draw calls would impact performance if redundant meshes or materials were imported into the final game engine scene.

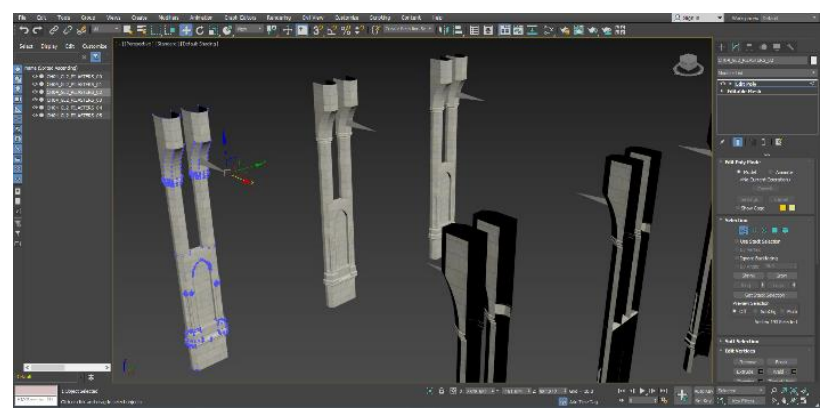

Figure 7. Correctly instanced components ready for Unity.

\subsection{Producing Materials}

It was quickly determined that the quality of default Revit textures was inadequate, and so different types of materials were produced for viewing in Unity 3D. There were broadly three different types of materials produced (figure 8):
1. Tiled materials using generic UV box-mapping

2. Render-to-texture materials with custom UV mapping

3. Photogrammetry materials.

The software Substance Designer by Allegorithmic was used for generating high-quality textures for the first two types of materials. Created textures were saved as JPGs and applied to meshes in Autodesk 3DS Max. The texture scale was validated during application. Considering the need to reduce draw calls, it was necessary to use the fewest materials possible, for which Type 1 was ideal. Type 2 materials were produced by consolidating Type 1 materials through Autodesk 3DS Max's 'render-to-texture' toolset.
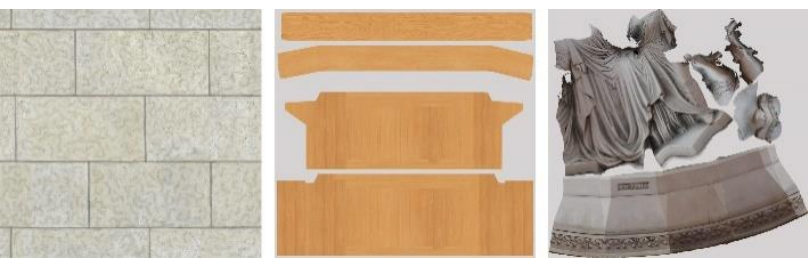

Figure 8 . Three different types of textures used in materials: 1 ) tiling 2) render-to-texture and 3) photogrammetric.

\subsection{Baking Normal Maps}

Meshes of smaller details - such as surface ornaments - had an extremely high polycount for their small scale. One can observe diminishing returns with regards to the amount of polycount required to represent these relatively small geometric detail accurately to an audience. Special efforts were therefore made to reduce the impact of these components on performance while maintaining their visual fidelity. A practice of 'normal map baking' - a technique common to the videogame industry - was used to store the appearance of geometric information in textures which respond to light and shadow realistically even if the underlying mesh lacks this detail. For this method, a low-detail version of the mesh was produced, either modelled manually in Rhinoceros 3D or Autodesk 3DS Max, or using the 'InstaLOD' plugin for Autodesk 3DS Max. Similarly to Type 2 textures they are created through Autodesk 3DS Max's 'render-to-texture' toolset. Figure 9 shows the comparison between an object before and after the conversion of geometry into a normal map.

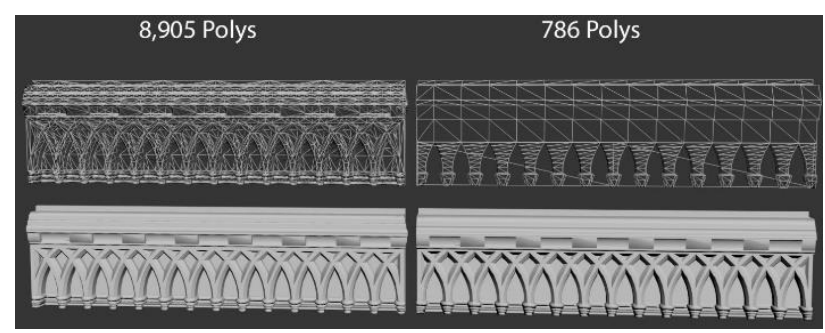

Figure 9. Comparison of a detail element with normal map.

\subsection{Processing Photogrammetry}

Photogrammetric models were used for particular components of high heritage value. Due to the thorough documentation of the Centre Block by HCS and CIMS, most significant heritage elements were available in a high-quality mesh. The photogrammetric meshes, however, required optimization for import into game engines. Photogrammetry was processed in Photoscan by Agisoft using the software's built-in tools for basic mesh repair and decimation. High and low poly versions of the photogrammetric meshes were exported so that geometric detail 
could be rendered to normal maps. Two methods of photogrammetric data used, one that followed the procedure listed above and an alternative that allowed for the manually modelling of a mesh with a very low polycount and the reprojecting of photogrammetric textures onto it (figure 10).

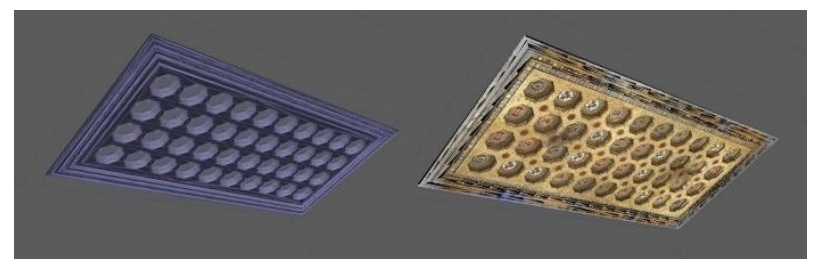

Figure 10. In LOD1.5 photogrammetry textures are applied to a modelled mesh as seen here with the Senate Ceiling.

These two methods were termed LOD2 and LOD1.5 respectively as shown in figure 5 (Bille et al., 2018). In either case, photogrammetric textures generally needed small touch-ups in Adobe Photoshop to correct visual errors and occluded geometry. Meshes were then finalized in 3DS Max to ensure correct scale and position, applied materials, and that any unnecessary faces were deleted. In addition to the diffuse albedo texture, normal, and occlusion maps were generated with the aformenttioned render-to-texture method and applied to the simplified mesh to preserve the detailed appearance of the high-poly photogrammetric mesh, while maintaining a low-poly result (figure 11).

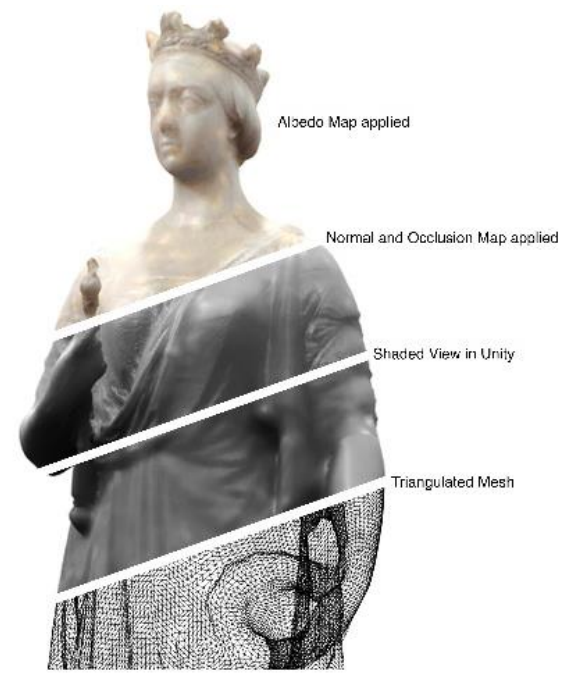

Figure 11. Photogrammetric mesh of a heritage statue with texture layers visible.

\subsection{Consolidating the Scene}

Considering the various software that the components pass through, it became vital to consolidate the scene in a software with reliable interoperability with game engines, such as Autodesk 3DS Max. During consolidation, all .FBX components are imported in an Autodesk 3DS MAX file and assigned correct JPG textures, created in Substance Designer. Components are then exported in .FBX format with separate JPG textures for import to Unity $3 \mathrm{D}$.

\subsection{Import to Unity and Viewing in VR}

4.6.1 Import to Unity3D: With all previous steps executed correctly, importing to Unity3D is a simple process. The tasks within pertain to folder structure and material assignments. With the high volume of components in the scene, a rigorous naming convention with meshes and materials was essential to avoid errors in Unity 3D. A clear folder hierarchy was defined for the scene, with each individual .FBX file receiving its own project folder containing its material and textures. The model components are individually added to the scene. It was essential the imported models be at the physically correct scale in order to be perceived correctly in VR. Type 1 materials are gathered in a shared folder so the same material can be applied to multiple meshes to optimize performance. Type 2 and 3 materials are stored with their respective meshes. One must be mindful of creating an efficient lighting environment for VR to perform correctly, meaning that all lighting in the scene should be prerendered 'baked lighting,' as the total number of draw calls will be multiplied by each dynamic light in the scene. Baked lighting, as seen in figure 12 within the Senate Chamber, is a rendering process that must be computed in advance of viewing the scene, and updated when the scene is changed.

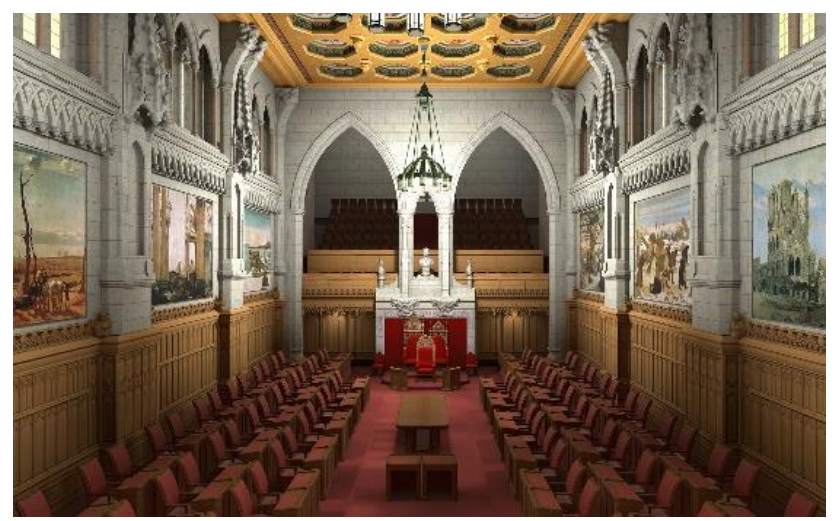

Figure 12. The Senate Chamber viewed in the Unity Editor with baked lighting.

4.6.2 VR Interface Setup: To view the scene, a pre-made VR camera controller is used to determine the position of the viewer. Toggling between different relevant viewpoints is permitted with the addition of a custom script. Finally, the scene was packaged as a standalone final build, making the scene viewable on any computer by running an .EXE file.

\section{DISCUSSION}

\subsection{Tracking Polycount}

Polycount is the most challenging concern in exporting geometry from Revit. The Oculus developer documentation states that the maximum range for effective performance in VR is between one and two million triangular faces. In comparison, exporting a mesh of the Library of Parliament from Revit without postprocessing produces a mesh of over 44 million triangular faces. As each .FBX file was prepared, the total polycount was tracked in a spreadsheet, such that if the total limit was surpassed, it was clear which files needed to be reduced further. Consequently, creating the .FBX files was an iterative process where individual files were re-meshed as the overall scene took shape. Following post-processing, the final polycount of the Library of Parliament was 1.53 million. To reduce the overall polycount of the Library of Parliament from 44 million to 1.53 million, decisions over where and what in the scene should be at a high detail versus low detail were made. 


\begin{tabular}{|c|c|c|c|c|c|}
\hline \multicolumn{2}{|c|}{ Direct Export from Revit } & \multicolumn{3}{c|}{ Final Game-Ready Meshes } \\
\hline $\begin{array}{c}\text { family } \\
\text { instances }\end{array}$ & $\begin{array}{c}\text { poly } \\
\text { count }\end{array}$ & $\begin{array}{c}\text { file size } \\
\text { MB }\end{array}$ & meshes & $\begin{array}{c}\text { poly } \\
\text { count }\end{array}$ & file size \\
\hline 15,070 & $\begin{array}{c}44.76 \\
\text { million }\end{array}$ & 310.63 & 9,483 & $\begin{array}{c}1,54 \\
\text { million }\end{array}$ & 87.23 \\
\hline
\end{tabular}

Figure 11. Polycount for the Library of Parliament comparing polycount from direct export from Revit against the workflow.

\subsection{A Stage for Storytelling}

The finished project produced by CIMS was a six-room virtual reality stage of the Centre Block of Canada, with no narrative to guide the visitor. When wearing a headsetsuch as an Oculus Rift or HTC Vive, the virtual visitor is transported to the virtual recreation of the House of Commons and Senate Chambers and Foyers, the Rotunda, and the Library of Parliament. They are free to walk around and examine the architectural details of the space. They have complete agency over their experience; however, they have no guide or narrative to direct their attention and keep their focus. According to Michael Mateas (Mateas and Stern, 2005), too much freedom and no limitations on how a virtual experience is explored can have a detrimental effect on the sense of presence and enjoyment of the experience. The translation of the BIM to VR provides an empty shell that must have additional content added to engage the virtual visitor and give a purpose to the time spent in virtual reality.

For the current project, CIMS role was to create the stage for a future curated experience. Due to a tight project timeline, the development of the virtual space and the creation of the narrative were developed concurrently. The specificities of the narrative to be added were not fully known while modelling occurred, making the requirements for the conversion from BIM to VR to ensure that the entire space was developed to a high quality. The specific point of views were identified; however, the intended direction of the gaze of the virtual visitor, potentially controlled by the future narrative, was not defined.
The lack of a defined narrative at the time of development caused issues with determining where the polygon count could be sacrificed and where it was crucial. Although this issue was resolved with an increase in communication with the team developing the narrative, some geometry within the space could have been modelled at a simplified geometry if the stage and narrative were not developed concurrently.

\section{CONCLUSION}

The BIM of the Parliament Hill National Historic Site is a highly detailed model that warrants dissemination through visualizations and immersive experiences. CIMS sought to leverage the benefits of this carefully made digital archive and convert the BIM into a VR experience that would allow the virtual visitor to explore the heritage spaces of the Centre Block of Canada, including the House of Commons and Senate Chambers and Foyers, the Rotunda, and the Library of Parliament. Through a complex workflow including Rhinoceros 3D and 3DS Max, CIMS was able to convert the model into one that conformed to the Oculus requirements for an optimized VR experience. Realistic textures were created using a combination of generic and unique textures generated from the documentation data. The result (Figure 12) is a fully operational VR experience that can be explored through an Oculus Rift headset. When made available to the public, visitors will be able to enjoy the Parliament Buildings virtually during their closure.

\section{ACKNOWLEDGEMENTS}

CIMS would like to thank the following people for their invaluable efforts in this project and preparing this paper: Dan Alphonza, Silvia Blanco-Pons, Berta Carrión-Ruiz, Josh Chartrand, Lara Chow, Rubin de Jonge, Abhijit Dhanda, Shawn Duke, Michael Duric, Andrée-Anne Godin, Tyler Grunt, Ayah Hassan, Petros Kapetanakis, Yana Kigel, Julia Laninga, Chiara Midali, Stephanie Murray, Raechel Padua, Hilary Romaniuk, Rehab Salama, Shane Shuster, Laurie Smith, Lauren Stoymennoff, Kyle Tousant, Kristi VanDelft, Josh Wallace, Adam Weigert, Remi Yuan, and Diego Zambrano.

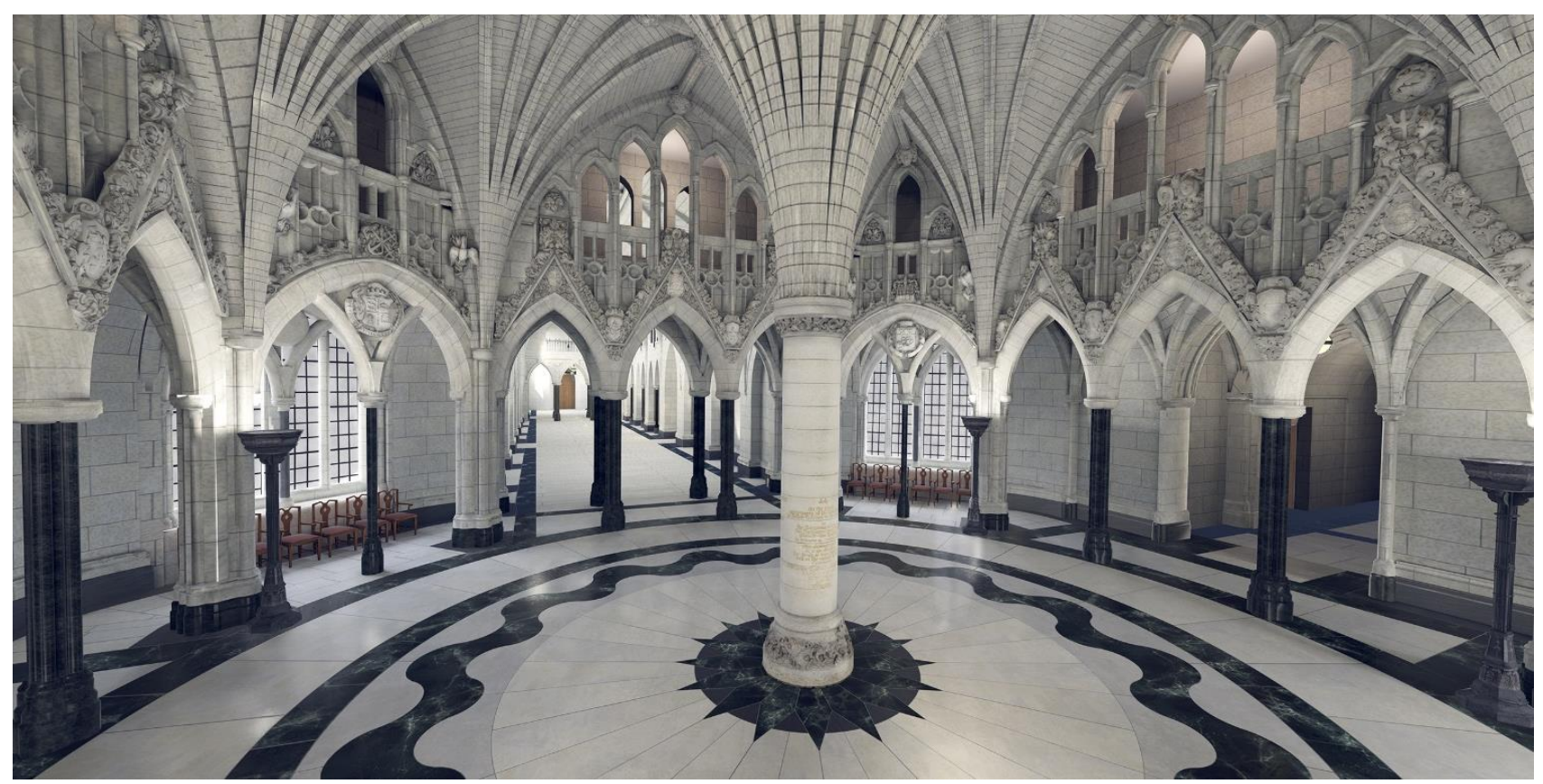

Figure 12: Final VR experience of the Rotunda. 


\section{REFERENCES}

Adobe,

2019.

Photoshop

CC.

https://www/adobe.com/photoshop

Adobe, 2018. Substance 2018. https://www.substance3d.com.

Advent, 2019. Advent Twinmotion 2019. https://www.twinmotion.com.

Agisoft, 2018. Photoscan 1.3.1. https://www.agisoft.com.

Autodesk, 2017. Autodesk 3DsMax 2017. https://www.autodesk.ca/en/products/3ds-max/

Bille, R., Smith, S.P., Maund, K., Brewer, G., 2018. Extending Building Information Models into Game Engines, In: Proceedings of the 2014 Conference on Interactive Entertainment. ACM, pp. 130-145.

Brooks, J., Frederick, 1987. Walkthrough-a dynamic graphics system for simulating virtual buildings, In: Proceedings of the 1986 Workshop on Interactive 3d Graphics. ACM, pp. 9-21. https://doi.org/10.1145/319120.319122.

Dhanda, A., Ortiz, M.R., Weigert, A., Paladini, A., Min, A., Gyi, M., Su, S., Fai, S., Santana Quintero, M., 2019. Recreating Cultural Heritage Environments For Vr Using Photogrammetry. In: The International Archives of the Photogrammetry, Remote Sensing and Spatial Information Sciences, Vol. XLII-2-W9, pp. 305-310.

Du, J., Zou, Z., Shi, Y., Zhao, D., 2018. Zero latency: Real-time synchronization of BIM data in virtual reality for collaborative decision-making. In: Automation in Construction, Vol. 85, pp. 51-64. https://doi.org/10.1016/j.autcon.2017.10.009.

Eliashvili, K., 2016. An assessment of the Industry Foundation Classes OpenBIM Format for Cross-Platform Building Information Modelling Workflows for Architectural Heritage. Thesis from Dublin Institute of Technology.

Enscape, 2019. Enscape. https//enscape3d.com.

Graham, K., Pybus, C., Arellano, N., Doherty, J., Chow, L., Grunt, T., Fai, S., n.d. Defining Geometry Levels to Optimize BIM for VR: Insights from Traditional Architectural Media. In: Technology Architecture Design. forthcoming.

Held, R.M., Durlach, N.I., 1992. Telepresence. In: Presence: Teleoperators Virtual Environments, Vol. 1, pp. 109-112. https://doi.org/10.1162/pres.1992.1.1.109.

InstaLOD GmbH, 2018. InstaLOD Software. https://instalod.com

IrisVR, 2019. IrisVR. https://irisvr.com.

Lachambre, S., Lagarde, S., Jover, C., 2017. Photogrammetry Workflow. Unity. https://unity.com/solutions/photogrammetry.

Lehtinen, S., 2002. Visualization and Teaching with State-of-theArt 3D Game Technologies, In: Connecting the Real and the Virtual - Design e-Ducation, 20th ECAADe Conference Proceedings. Warsaw, Poland, pp. 538-541.
Mateas, M., Stern, A., 2005. Interaction and Narrative, in: Salen, K., Zimmerman, E. (Eds.), The Game Design Reader: A Rules of Play Anthology. MIT Press, Boston, MA, pp. 642-669.

Minsky, M., 1980. Telepresence. In: Omni, pp. 45-51.

Nandavar, A., Petzold, F., Schubert, G., Ag, G., 2018. Interactive Virtual Reality Tool For Bim Based On Ifc - Development of OpenBIM and Game Engine based layout planning tool - a novel concept to integrate BIM and VR with bi-directional data exchange. In: CAADRIA 2018 Proceedings, pp. 453-462.

Novitski, B.J., 1998. Rendering Real and Imaged Buildings: the Art of Computer Modeling from the Palace of Kublai Khan to Le Corbusier's Villas. Rockport Publishiers, University of Michigan.

Oculus Documentation, n.d. Guidelines for VR Performance Optimization.

https://developer.oculus.com/documentation/pcsdk/latest/conce pts/dg-performance-guidelines/ (11 January 2019).

Pybus, C., 2019. New Tools For Cultural Heritage Tourism: Accessible Virtual Reality For Milan's Basilica Sant'Ambrogio. In: ISPRS - International Archives of the Photogrammetry, Remote Sensing and Spatial Information Sciences. pp. 10031010.

Pybus, C., 2018. Intangible Territories: The Island of Salty Dreams. Thesis from Carleton University.

Rheingold, H., 1991. Virtual reality. Summit Books, New York.

Robert McNeel and Associates, 2017. Rhinoceros 3D 5. https://www.rhino3d.com/

Sheridan, T.B., 1992. Musings on Telepresence and Virtual Presence. In: Presence: Teleoperators Virtual Environments, Vol. 1, pp. 120-126. https://doi.org/10.1162/pres.1992.1.1.120.

Slater, M., Usoh, M., 1993. An Experimental Exploration of Presence in Virtual Environments. In: Department of Computer Science Technical Reports: up to 1999. https://qmro.qmul.ac.uk/xmlui/handle/123456789/4705

Steuer, J., 1992. Defining Virtual Reality: Dimensions Determining Telepresence. In: Journal of Communication, Vol. 42, pp. 73-93. https://doi.org/10.1111/j.14602466.1992.tb00812.x.

Unity Technologies, 2018. Unity 3D 2018. https://unity3d.com/

Yan, W., Culp, C., Graf, R., 2011. Integrating BIM and Gaming for Real-Time Interactive Architectural Visualization. In: Automation in Construction, Vol. 20, pp. 446-458.

Zaker, R., Coloma, E., 2018. Virtual reality-integrated workflow in BIM-enabled projects collaboration and design review: a case study. In: Visualization in Engineering, Vol 6 (1), pp. 1-15. https://doi.org/10.1186/s40327-018-0065-6.

Zeltzer, D., 1992. Autonomy, Interaction, and Presence. In: Presence: Teleoperators Virtual Environments, Vol. 1, pp. 127132. https://doi.org/10.1162/pres.1992.1.1.127. 